\title{
A New Paradigm for Train to Ground Connectivity Using Angular Momentum
}

\author{
Ben H. Allen \\ Department of Engineering Science \\ University of Oxford \\ Parks Road \\ Oxford, OX1 3PJ, UK \\ ben.allen@eng.ox.ac.uk
}

\author{
Tim W. C. Brown \\ Institute for Communication Systems, \\ University of Surrey \\ Guildford, GU2 7XH, UK \\ tim@brown76.name
}

\author{
Tim D. Drysdale \\ School of Engineering \\ University of Edinburgh \\ Edinburgh, EH8 9YL, UK \\ timothy.drysdale@ed.ac.uk
}

\begin{abstract}
Wireless connectivity needs to be high capacity and reliable for present and future high speed land transportation systems, including rail, road and hyperloop. All such moving platforms transport a substantial number of passengers, creating high data demands in often remote areas. This paper proposes how to re-think the means by which wireless connectivity is implemented to provide wireless services to a dense number of users on a fast moving platform. The proposed concept evolves from orbital angular momentum radio modes for data multiplexing to linear or planar angular momentum instead. In this study, the wireless link is placed underneath the moving platform, a promising low-cost method that potentially could achieve $9 \mathrm{Gbps}$ with $15 \mathrm{MHz}$ bandwidth and $10 \mathrm{~dB}$ signal to noise ratio from initial analysis carried out.
\end{abstract}

\section{Keywords-Angular Momentum, MIMO, Rail Communication}

\section{INTRODUCTION}

Trains can attract in excess of 3000 passengers, whereby the majority are earnestly seeking connectivity to the Internet or telephone network. The intense number of users of mobile services within a confined space have to share the limited communication resources from an external wireless link due to: high demand; substantially high Doppler shifts; the high penetration loss and also the high vulnerability to co-channel interference between users within a train carriage. These, among other factors, limit the connectivity such that there is an inconsistency in delivered data rates to a user, as well as call drops over the course of a journey. Coverage is further limited by the need to provide backhaul from the nearest available fibre link.

Current rail connectivity has adopted trackside/remote base stations [1] and in-built antennas or leaky feeders within tunnels [2][3]. Radio-over-fibre is seen as another possible candidate [1]. These technologies involve a wireless link from a fixed point that is propagating some given distance and the change in channel state is rapid, which adds significant "stress" on the system to deliver highly demanding data rates. A recent study in the United Kingdom reveals that the average expected data requirements per user on a train could increase to $3 \mathrm{Mbits} / \mathrm{s}$ beyond 2025 [4]. Hence a data rate in the order of $9 \mathrm{Gbits} / \mathrm{s}$ is required for a train of 3000 passengers. Additional data requirements within the train's own infrastructure include on-board sensors [5], closed circuit television links and other auxiliary data [6]. Using trackside base station masts to deliver these services with mmWave broadband connection is one possibility, where the inter-site distance is expected to be less than $1 \mathrm{~km}$ [7]. Implementation of the required number of masts is costly and their deployment in rural areas could prove impractical, especially in areas of outstanding natural beauty. There is a substantial need for a robust, low cost and low profile reliable infrastructure to connect trains. The technology could also apply to other fast moving platforms including the possible hyperloop and road vehicles. Any available frequency of operation could be selected, which may include bands licensed for other purposes due to the limited and contained propagation range.

This paper presents the concept of evolving the promising method of Orbital Angular Momentum (OAM) [8], to a new concept formed by the authors defined as Linear (LAM) and Planar (PAM) Angular Momentum. It demonstrates for the first time how an example implementation of LAM could form a mobile connection between the underside of a train carriage and the sleepers on the track such that the pre-coded propagation channels could theoretically exceed the required data demands for future railways.

\section{EVOLUTION OF ORBITAL TO LINEAR AND PLANAR ANGULAR MOMENTUM}

It is well known that OAM radio has several ways in which an antenna can be built to generate the different modes from which data can be multiplexed. One commonly known implementation as illustrated in Fig. 1 (a) is to use a uniform circular array [9]. In this example six elements are used and if two such arrays were facing each other and using a lens at each end of the link would prevent the transmitted antenna pattern from dispersing [10], there are six possible orthogonal modes that can be used as additional communications resources $( \pm 1 \ldots \pm 3)$. Since antenna arrays are used in this instance, this link is essentially a 6x6 Multiple Input Multiple Output (MIMO) link, but the antenna element topology at each end is arranged in such a way as to exploit the degrees of freedom in the orbital domain around the direction of propagation.

Where OAM is implemented with discrete elements as a $N \times N$ MIMO link in this way, it is well known that there is the possibility to produce six angular modes where mode $n$ is defined by the following equation when radiating at the boresight of the transmit antenna and aligned with the boresight of the receiver [11]:

$$
\text { Mode } n=\sum_{i=1}^{N} e^{j \frac{n i 2 \pi}{N}}
$$


Additionally, for mode $n$, the $i^{\text {th }}$ element will have a phase weight equal to $n i 2 \pi / N$. It is possible to evolve OAM into PAM as illustrated in Fig 1 (b) in a rectangular planar form and to LAM in a linear form in Fig. 1 (c). Provided the elements are sufficiently spaced apart, LAM and PAM can generate the same six or $N$ modes. For OAM, lenses overcome dispersion, while LAM and PAM can use suitable antenna pattern design to reduce the required element spacing but they are more tolerant of misalignment than LAM. Though PAM is possible, LAM will be studied in this paper as a more practical application for railway communications which could be achieved from the side of a train, but the underneath of a train will be considered in this paper.

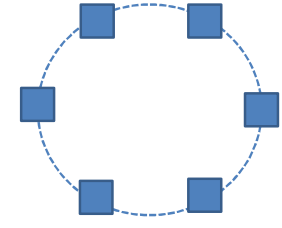

(a)

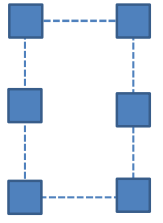

(b)

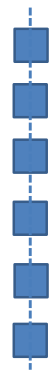

(c)
Fig. 1 Evolution of a six element (a) orbital angular momentum to a (b) planar and (c) linear angular momentum.

Fig. 2 illustrates the dimensions used in LAM where the antenna elements on the track sleepers and the rolling stock underside are directly facing. The propagation distance is determined by vertical distance $d_{z}$, with element separation $d_{x}$. Each element has a directive antenna beam pattern and it is necessary to separate the antenna elements such that either there is low cross-coupling of fields to the neighboring elements; or that if there is, the spacing is organized such that the links are spatially orthogonal. The latter case would be forming a setup akin to line-of-sight spatial multiplexing in MIMO, but LAM goes a step further by including the angular characteristics and it is further possible to include polarization as another degree of freedom. For example, the antennas could alternate with vertical and horizontal polarization to further reduce the cross-coupling.

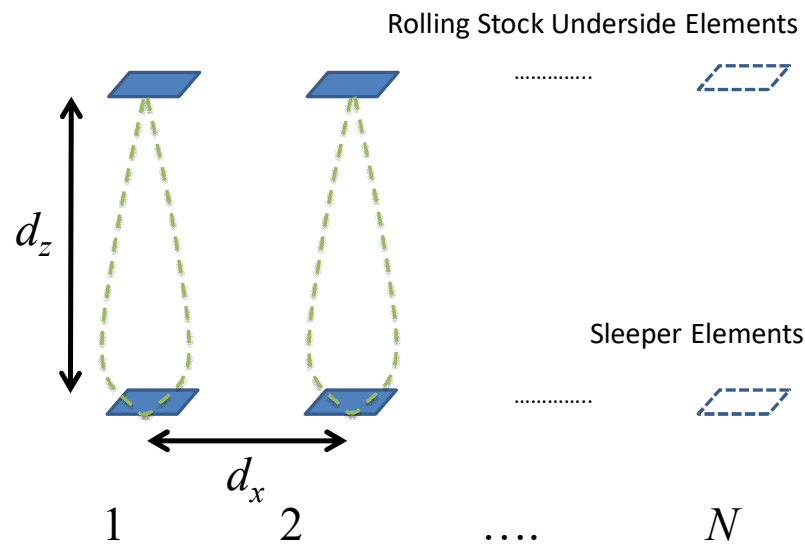

Fig. 2 Illustration of a LAM scenario with a moving train when the transmit and receive elements are facing.
To analyze how the antenna beamwidth affects the required antenna element separation, the channel capacity is plotted versus the ratio $d_{x} / d_{z}$ and for when $N=2$, as shown in Fig. 3. A Signal-to-Noise Ratio (SNR) of $10 \mathrm{~dB}$ is assumed. The capacity is calculated by determining the $2 \times 2$ MIMO channel matrix $\mathbf{H}$ through simple ray tracing, from which the Shannon limit can be easily resolved [12]. The two LAM modes derived in equation (1) in this instance are equal to the precoder that would be applied to the MIMO channel to achieve such a capacity. A beamwidth of $60^{\circ}$ is compared with a narrower beamwidth of $10^{\circ}$. For the narrow beamwidth, it can be seen that the capacity rapidly rises to twice the SISO capacity when $d_{x} / d_{z}=0.25$. At this point, the antenna elements have moved sufficiently outside of the beamwidth between their neighboring elements that there is negligible cross-coupling and so two orthogonal MIMO subchannels with LAM precoding have been formed. Inevitably, for the wider beamwidth, the antenna elements need to separate substantially further beyond $d_{x} / d_{z}=1$ in order for the capacity limit to be reached. However, at closer distances such as $d_{x} / d_{z}=0.35,0.6$, and 0.8 , it is observed that the capacity reaches the peak at these points. These are cases where the antenna elements are spaced such that the channel $\mathbf{H}$ is spatially orthogonal, which is possible as the beamwidth of the antennas are wide enough to allow all four channel coefficients to have near equal magnitude.

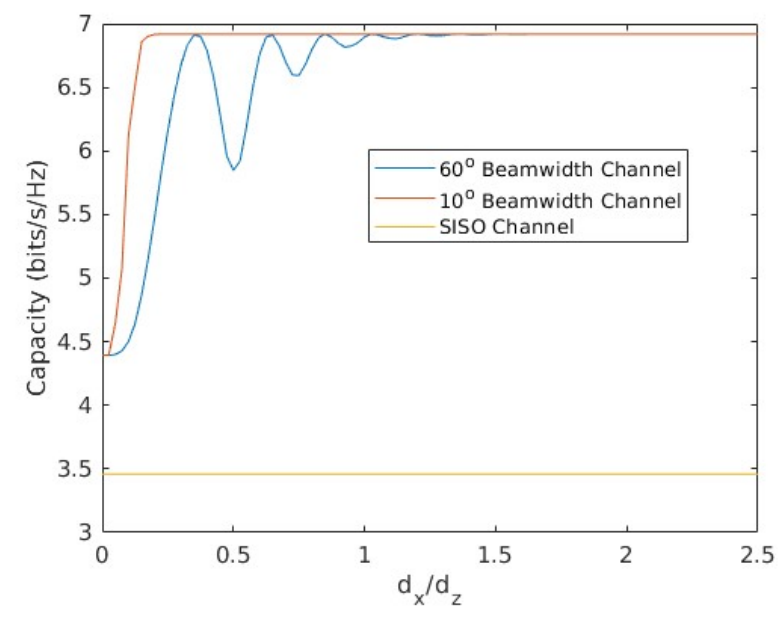

Fig. 3 Plot of the capacity for $10 \mathrm{~dB}$ SNR achieved for elements facing versus the ratio of spacing $d_{x} / d_{z}$ for antenna beamwidths of $10^{\circ}$ and $60^{\circ}$.

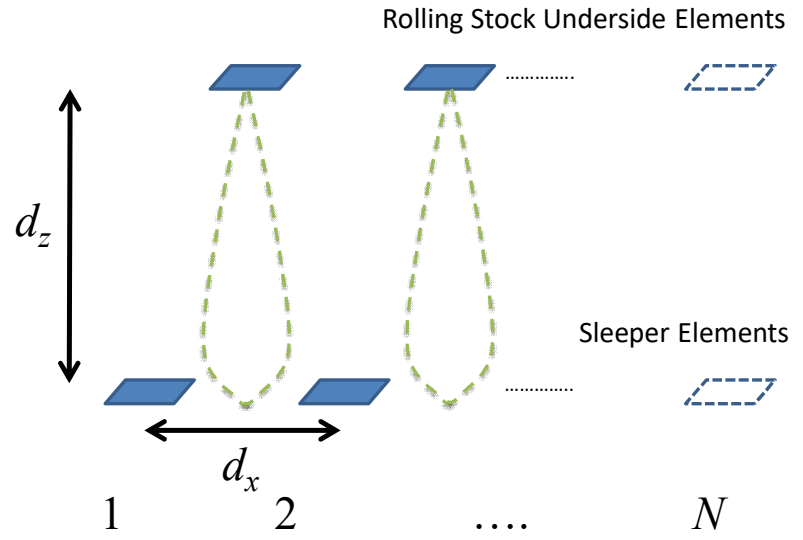

Fig. 4 Illustration of a LAM scenario with a moving train when the transmit and receive elements are offset. 
In the case of a wireless link underneath a train employing LAM, movement will cause the antenna elements to become offset compared to those along the track, as shown in Fig. 4. This will inevitably degrade the capacity as this happens because the links between facing antennas will weaken while the cross-coupling will increase. It is at this point that LAM is required to take another further step beyond spatial multiplexing and deploy the antenna elements in a way that will create a spatial channel such that as the train moves, there is a seamless wireless connection that has minimal time variant change and therefore minimal Doppler spread [13]. Though different solutions to this will exist, one solution that is proposed is illustrated in Fig. 5 (a) to (c), which are taken as a top view assuming the floor of the train carriage is transparent. The red, blue and grey diagonal solid lines moving upwards represent three snapshots from (a) to (c) of two moving antenna elements attached to the train. Just two of the train's antenna elements, each with switchable polarization, are shown for clarity. The horizontal antenna elements on the track are attached to the sleepers represented by the dashed horizontal lines, which are blue and red to represent vertical and horizontal polarization respectively.

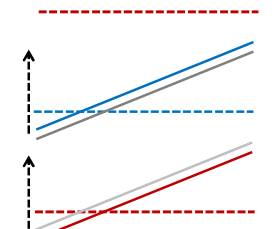

(a)

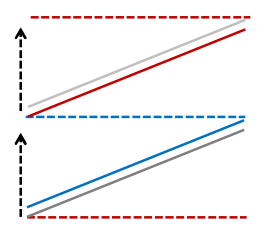

(b)

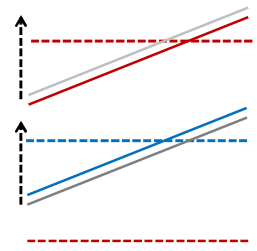

(c)
Fig. 5 Illustration of a LAM scenario with two elements on the train showing (a) initial position and (b) position where polarizations switch over and (c) new position after switchover. Blue has vertical polarization and red has horizontal polarization. Grey elements represent inactive polarizations. Horizontal lines represent the track antennas with alternating polarization.

At the first position in Fig. 5 (a), the upper antenna is switched to vertical polarization covering a vertically polarized sleeper. The lower antenna is likewise switched to horizontal polarization covering a horizontally polarized sleeper. In Fig. 5 (b) the train has moved to a position such that its two antennas are in between two sleepers. At this point the upper antenna has switched from vertical to horizontal polarization. Likewise the lower antenna has switched from horizontal to vertical polarization. This finally allows the train to move over the next pair of sleepers with matching polarizations as seen in Fig. 5 (c). This switching mechanism repeats as the train moves over further sleepers with alternating polarization. Arranging the polarizations in this manner will ensure that interference between neighboring antenna elements will be minimized. Polarization switching will require the LAM modes to be programmed to move at the velocity of the train, which will be known. Likewise the train will pre-code the required phases on its antennas for LAM while the sleeper antennas only require fixed cophasing. This ensures all the communication effort is kept on board the train and minimized on the track, which need only activate the relevant antenna elements and transceivers as the train is approaches.

\section{CAPACITy ANAlysis FOR High SpeEd TRAins}

In section II it has been established how LAM could be deployed on a railway track in order to maintain a seamless connection. If the environment around the track was complete free space, the channel would be entirely stationary and would have no Doppler spread. However, the train's wheels, the track, ballasts and other infrastructure underneath the train are vulnerable to causing reflections from the transmit to the receive elements. This would cause the channel to have a Ricean distribution with a line of sight component that has no Doppler shift [13]. Designing the antennas to have specifically designed radiation patterns can, for such a short propagation distance, suitably craft the propagation channel to have a high Rice factor by not allowing the antenna patterns to have high gains in undesired directions, i.e. low side-lobe levels. Based on the assumption of Ricean fading, this section evaluates the maximum ideal capacity using LAM for up to ten train carriages.

For the example case it is assumed that $d_{z}=0.5 \mathrm{~m}$ and that antennas with a beamwidth of $60^{\circ}$ are used. As noted from Fig. 3 , it is more than sufficient if $d_{x}=1.2 d_{z}$ to reach the capacity limit if all elements are uniformly spaced. Therefore $d_{x}=$ $0.6 \mathrm{~m}$. It is assumed that the example train has a carriage length of $20 \mathrm{~m}$ where antenna elements can be deployed in an ideal sense and therefore 26 elements per carriage are achievable allowing about $2 \mathrm{~m}$ clearance each end of the carriage. In practical cases using the whole carriage length may not be possible. Additionally, it is assumed the switching in Fig. 5 is fully synchronised with the velocity of the train. From this, using a stochastic channel $\mathbf{H}$ where $i=1$ to $N$, elements $h_{i i}$ will have independent Ricean distributions all of equal Rice factor but the cross coupling to all other elements $h_{i j}(j \neq i)$ can be assumed to be zero. From this channel model, the Shannon capacity limit for different numbers of carriages and Rice factors can be determined.

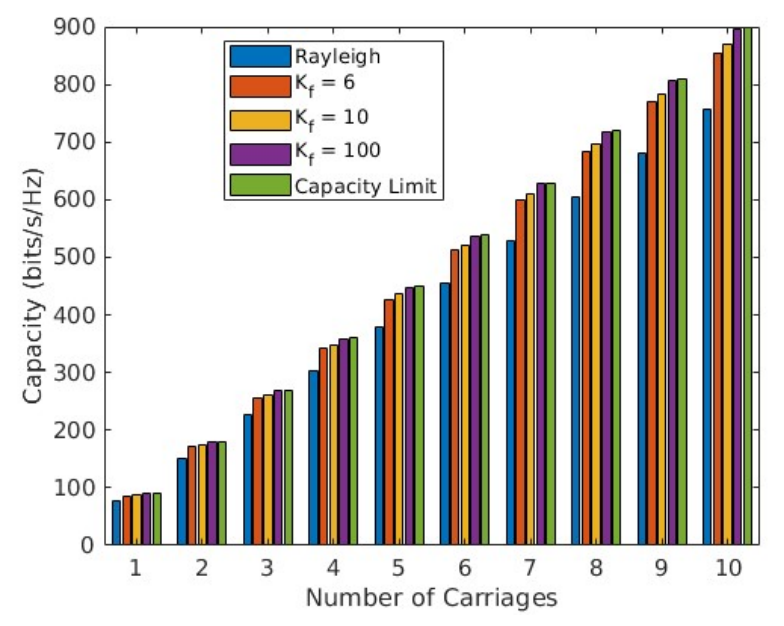

Fig. 6 Average capacity dependent on the number of carriages and the Rice factor of the channel against an ideal case with infinite Rice factor. SNR = $10 \mathrm{~dB}$

Fig. 6 plots a bar chart of the average capacity vs number of carriages for Rice factors of zero, 6, 10 and 100. It should be noted that for a Rice factor of zero, this is equal to a Rayleigh distribution with no line-of-sight [13] and therefore will never occur. However, it is shown here as a lower limit. 
Results show that for Rice factors even as low as 6, which are a more realistic worst case scenario, it is still possible to achieve over $90 \%$ of the capacity limit.

As well as the average capacity, it is also useful to analyse the distribution, where for the case of 10 carriages it is plotted in Fig. 7. Note that the curve for a Rice factor of 100 cannot be seen because it is covered by the capacity limit line. Where there are fewer carriages, it is the case that the capacity curves would still have the same characteristic, only offset to lower average values, as found in Fig. 6. It is clear that as the Rice factor rises above 6 , the capacity is near constant.

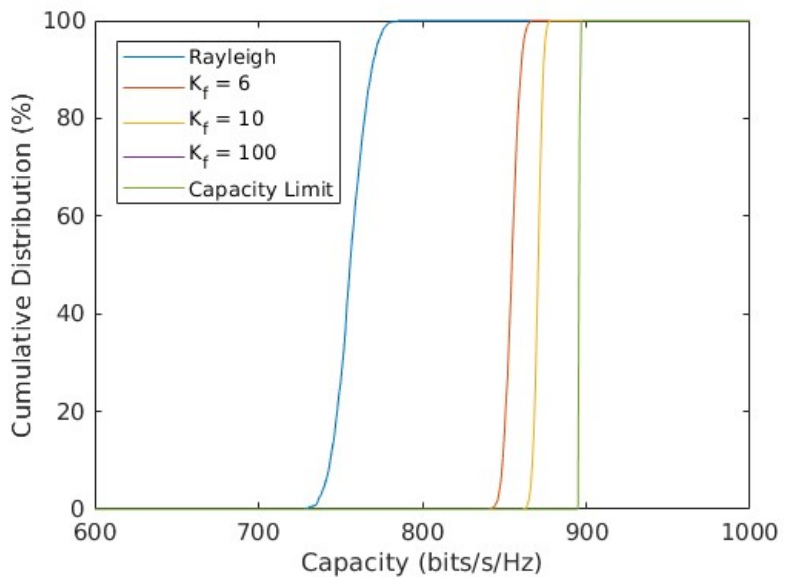

Fig. 7 Cumulative distribution of the Rice factor for the case of ten carriages and varying Rice factor. Note - The Rice factor of 100 has the same distribution as the capacity limit. $\mathrm{SNR}=10 \mathrm{~dB}$

Referring to the capacity requirements at the start of this paper where it is expected that a ten carriage train may reach data capacity requirements in the order of $9 \mathrm{Gbits} / \mathrm{s}$, then for a ten carriage train, a system bandwidth of $10-15 \mathrm{MHz}$ could be adequately meet this requirement accounting for guard bands and waveform limitations. This is on the assumption that the SNR is at the minimum expected level of $10 \mathrm{~dB}$, though a higher SNR could further reduce this bandwidth. However energy consumption would be a limitation in this regard. Other practical obstacles would need to be taken into account where propagation is affected by undesirable items covering the antennas on the track such as leaves, dirt and ballasts. A system that can exceed the desired capacity would be required to allow contingency for such instances.

\section{ACKNOWLEDGMENT}

Ben Allen is grateful for the support of the Royal Society Industrial Fellowship scheme under award number [IF160001].

\section{CONCLUSION}

A new paradigm for communicating with trains and long fast moving transportation platforms has been presented in this paper, where short wireless links are possible. Track to underneath the train is proposed here in order to move to ultrafast ultra-reliable communication. The concept of OAM radio multiplexing has been evolved to PAM and LAM, which could be arranged in a suitable way so as to craft the propagation channel to be seamless and have a suitable level of stationarity that maintains a reliable link. Results show that a small bandwidth has the capability to meet the current and future capacity requirements on board a train. Implementation is low cost, low profile, robust and has low energy consumption while it can be implemented within a chosen frequency band.

\section{REFERENCES}

[1] D. T. Fokum and V. S. Frost, "A Survey on Methods for Broadband Internet Access on Trains", IEEE Communications Surveys \& Tutorials, vol. 12, no. 2, pp. 171-185, Second Quarter 2010.

[2] K. Guan, Z. Zhong, J. I. Alonso and C. Briso-Rodriguez, "Measurement of Distributed Antenna Systems at $2.4 \mathrm{GHz}$ in a Realistic Subway Tunnel Environment", IEEE Transactions on Vehicular Technology, vol. 61, no. 2, pp. 834-837, Feb. 2012.

[3] C. Briso-Rodriguez, J. M. Cruz and J. I. Alonso, "Measurements and Modeling of Distributed Antenna Systems in Railway Tunnels", IEEE Transactions on Vehicular Technology, vol. 56, no. 5, pp. 2870-2879, Sept. 2007.

[4] "Advice to Government on improving rail passenger access to data services Spectrum for trackside connectivity solutions and rail passenger data demand", Office for Communications, UK, August 2018.

[5] M. Sneps-Sneppe and D. Namiot, "On 5G Projects for Urban Railways," 2018 22nd Conference of Open Innovations Association (FRUCT), Jyvaskyla, 2018, pp. 244-249.

[6] B. Allen et al. "User and System Requirements", X2Rail Deliverable D3.1, Project Call H2020-S2RJU-CFM-2015-01-1, Grant Number 730640, September 2016.

[7] S. Friedner, "5G Infrastructure Requirements in the UK", Report for the National Infrastructure Commission, LS Telecom, Version 3.0, December 2016.

[8] B. Allen, D. Simmons , T. D. Drysdale and J. Coon, "Performance analysis of an orbital angular momentum multiplexed amplify-andforward radio relay chain with inter-modal crosstalk", Royal Society Open Science, vol. 6, issue 1, January 2019.

[9] T. D. Drysdale, B. Allen, C. Stevens, S. J. Berry, F. C. Smith and J. Coon, "How orbital angular momentum modes are boosting the performance of radio links", IET Microwaves, Antennas \& Propagation, vol. 12, no. 10, pp. 1625-1632, 1582018.

[10] B. Allen et al., "Reduction of orbital angular momentum radio beam divergence using a 3D printed planar graded index lenses," 12th European Conference on Antennas and Propagation (EuCAP 2018), London, 2018, pp. 1-3.

[11] S. M. Mohammadi et al., "Orbital Angular Momentum in Radio-A System Study," in IEEE Transactions on Antennas and Propagation, vol. 58, no. 2, pp. 565-572, Feb. 2010.

[12] T. W. C. Brown, E. DeCarvalho, P. Kyritsi, "Practical Guide to the MIMO Radio Channel", Wiley, 2012, UK.

[13] S. R. Saunders, A. Aragón-Zavala, "Antennas and Propagation for Wireless Communication Systems”, Wiley, 2007, UK. 